\title{
Multilayer compression bandaging in the acute phase of deep-vein thrombosis has no effect on the development of the post-thrombotic syndrome
}

\author{
E. M. Roumen-Klappe $\cdot$ M. den Heijer $\cdot$ J. van Rossum • \\ H. Wollersheim - C. van der Vleuten - Th. Thien • \\ M. C. H. Janssen
}

Published online: 15 May 2008

(C) The Author(s) 2008

\begin{abstract}
Objective The purpose of this randomized study was to evaluate the influence of immediate multilayer compression bandages before application of elastic stockings in the acute phase of deep-vein thrombosis (DVT) on development of the post-thrombotic syndrome (PTS). Methods Sixty-nine patients with acute symptomatic DVT were randomized to immediate bandaging $(n=34)$ or no bandaging $(n=35)$. After reduction of edema sized-to-fit elastic stockings were applied in all patients after 7-14 days. Follow-up visits and non-invasive examinations were planned after 7, 30 and 90 days and 1 year. Venous outflow resistance (VOR) was measured by strain gauge plethysmography. Thrombosis score (TS) and reflux were
\end{abstract}

E. M. Roumen-Klappe · J. van Rossum · H. Wollersheim ·

Th. Thien · M. C. H. Janssen $(\square)$

Department of General Internal Medicine, University Medical

Centre St. Radboud, P.O. Box 9101, 6500 HB Nijmegen,

The Netherlands

e-mail: M.Janssen@aig.umcn.nl

M. den Heijer

Department of Epidemiology and Biostatistics, University

Medical Centre St. Radboud, P.O. Box 9101,

6500 HB Nijmegen, The Netherlands

M. den Heijer

Department of Endocrinology, University Medical Centre

St. Radboud, P.O. Box 9101, 6500 HB Nijmegen,

The Netherlands

H. Wollersheim

Centre of Quality of Care Research, University Medical Centre

St. Radboud, P.O. Box 9101, 6500 HB Nijmegen,

The Netherlands

C. van der Vleuten

Department of Dermatology, University Medical Centre

St. Radboud, P.O. Box 9101, 6500 HB Nijmegen,

The Netherlands measured by duplex scanning. After one year patients were evaluated for clinical PTS using both the clinical scale of the CEAP classification and the Villalta score. Results Improvement of clinical symptoms and decrease of leg circumference was better on day 7 in the bandaging group, but after 1 and 3 months clinical symptoms had improved equally in both groups. In 7 patients in the no-bandaging group a bandage was applied after all because of persistent edema after 10 days. There were no differences in VOR, TS and reflux. Using the CEAP classification the incidence of PTS was $39 \%$ in patients with bandages and $42 \%$ in patients without bandages (RR $0.91,95 \%$ CI $0.50-1.66$ ). Using the Villalta score the incidence of PTS was resp. 29 and 33\% (RR $0.87,95 \%$ CI $0.41-1.8$ ). There was no difference in severity of PTS. Conclusion Immediate multilayer compression bandaging in the acute phase of DVT is effective in reducing edema and complaints in the first week, but has no effect on thrombus regression, valve incompetence and the development of clinical PTS after 1 year.

Keywords Deep-vein thrombosis - Post-thrombotic syndrome $\cdot$ Compression bandaging

$\begin{array}{ll}\text { Abbreviations } \\ \text { PTS } & \text { Post-thrombotic syndrome } \\ \text { VOR } & \text { Venous outflow resistance } \\ \text { DVT } & \text { Deep-vein thrombosis } \\ \text { TS } & \text { Thrombosis score }\end{array}$

\section{Introduction}

Deep vein thrombosis (DVT) is a relatively common disease that can be associated with acute pulmonary embolism (PE), as well as the more chronic post-thrombotic syndrome (PTS). Although it was generally considered to take $5-10$ years to 
develop PTS, the current opinion is that PTS already becomes clinically manifest within 1-2 years following the acute thrombotic event [1-3]. Impairment of quality of life is correlated with severity of PTS $[4,5]$. The pathogenesis of PTS is not completely understood, but it is suggested that persistent obstruction and the development of valvular insufficiency after DVT leads to venous hypertension, resulting in symptoms like pain and swelling of the leg and signs like edema, hyperpigmentation and the occurrence of venous ulcers. In a previous study it was shown that the combination of an elevated venous resistance, thrombosis score and reflux 3 months after DVT could predict PTS with reasonable accuracy [2]. For this reason preventing valve damage and reducing venous hypertension are likely to be important in preventing PTS already early in the treatment of DVT.

Outpatient guidelines on treatment of DVT include patient counselling regarding low molecular weight heparin (LMWH) injections, oral anticoagulation, ambulation as tolerated and the use of compression stockings with a pressure of $30-40 \mathrm{mmHg}$ during 2 years after an episode of DVT. Evidence supporting the effectiveness of elastic compressive stockings (ECSs) comes from two studies that demonstrated that use of ECSs resulted in a decreased incidence of PTS $[1,6]$. As a result of these trials it has become common clinical practice to prescribe ECSs for patients with DVT. However, compression stocking therapy was delayed for 1-3 weeks, since it takes time to decrease leg swelling and to measure and produce the stockings. In continental Europe there is an old tradition to treat mobile patients who have acute DVT with multilayer compression bandages. The bandaging has to be done by specially trained nurses and is time-consuming while the evidence for this therapy remains scarce. Only one small study showed that immediate ambulation and compression therapy leads to faster relief of symptoms like pain and swelling and a reduction of PTS [7].

The hypothesis of this study was that immediate multilayer compression bandaging in the first 2 weeks of DVT is effective in reducing symptoms on the short term and PTS on the long term, by preventing valve insufficiency and stimulation of thrombus regression. To evaluate this hypothesis we performed a prospective randomized, unblinded study in which the effect of immediate multilayer compression bandaging was compared to no bandaging before application of ECSs on clinical symptoms, venous outflow resistance (VOR), thrombosis score (TS), reflux and development of clinical PTS after 1 year.

\section{Methods}

Between 2002 and 2004 all consecutive patients with symptomatic DVT, confirmed by compression ultrasound, were considered eligible for the study. The duration of symptoms ranged from 1 to 90 (mean 8 days). Exclusion criteria were previous DVT, pulmonary embolism, a short life expectancy ( $<2$ years), paralysis of the leg, pre-existing leg ulcers or signs of venous insufficiency, bilateral thrombosis and arterial insufficiency $(A B I<0.9)$. The study was part of the Post Thrombosis Study (results not published yet), in which the role of inflammation in the acute phase of DVT is investigated. For this reason patients were also excluded because of malignant disease, pregnancy or puerperium, use of anti-inflammatory medication or other severe medical illnesses. The study was approved by the local ethics committee and all patients gave informed consent. Distal DVT was defined as thrombosis of only the calf veins, thrombosis of the popliteal vein and higher was defined as proximal DVT.

Patients were treated on the outpatient clinic with LMWH (dose based on body weight) and vitamin $\mathrm{K}$ antagonists (acenocoumarol, INR 2-3) for 3 months. Patients were randomized to multilayer bandaging or no bandaging on the first day. Bandages were applied by specialized nurses on the outpatient department every 3-4 days. Over wadding and short stretch bandages were applied on the lower leg. Only in proximal thrombosis elastic bandages (Dauerbinde $12 \mathrm{~cm} \times 14 \mathrm{~m}$ ) were applied on the upper leg.

For distal DVT (over a layer of Tubiton and wadding) linelastic short stretch $(40 \mathrm{mmHg})$ bandages (Idealbinde $10 \mathrm{~cm} \times 10 \mathrm{~m}$ ) were applied on the lower leg. Patients wore these bandages day and night and these were changed every 3-4 days or when they were too loosened. For proximal DVT the lower leg was bandaged the same way, for thigh long stretch bandages (Dauerbinde $12 \mathrm{~cm} \times$ $14 \mathrm{~m}$ ) were used. These long stretch bandages were removed during the night. After reduction of edema sizedto-fit graded ECSs were applied in both groups after 7-14 days. All patients were equally mobile and encouraged to walk as much as possible, leg elevation was advised between ambulation. When patients were randomized to no compression bandaging but edema was persistent after 10 days, compression bandage was applicated after all. All patients were instructed to wear the stockings for at least 2 years.

Follow-up visits and non-invasive examinations were planned after 7, 30 and 90 days and 1 year. Clinical scores of symptoms were calculated and circumference of the leg was measured $12 \mathrm{~cm}$ above kneejoint, $12 \mathrm{~cm}$ under kneejoint and at the ankle. The difference of circumference between the thrombosed and non-thrombosed leg were measured each visit.

After 1 year patients were evaluated by a dermatologist who was unaware of the results of the venous examination. The severity of PTS was scored according to both the 
clinical score (range 0-6) of the revised CEAP classification (Clinical, Etiologic, Anatomic, and Pathophysiologic) [8] and the Villalta score [3]. In the CEAP classification patients with class 0 represent no visible or palpable signs of venous disease symptoms of PTS; class 1 telangiectases, reticular veins or malleolar flare; class 2 varicose disease; class 3 oedema without skin changes; class 4 skin changes ascribed to venous disease (4a eczema and pigmentation, $4 \mathrm{~b}$ lipodermatosclerosis and atrophy blanche); class 5 skin changes with a healed ulcer and class 6 skin changes with active ulceration. PTS was defined present if the classification was 3 or more. The Villalta score grades the severity from 0 to 3 of five symptoms (pain, cramps, heaviness, pruritus and paresthesia) and six signs (edema, skin induration, hyperpigmentation, venous ectasia, redness and pain during calf compression). A summed total score of $\geq 5$ indicates PTS, and $>14$ or presence of ulcer indicates severe PTS.

The VOR was measured in both extremities by straingauge plethysmography as described previously $[9,10]$. In summary the patients were examined in supine position with pneumatic cuffs around the thighs and strain gauges around the calves. After inflation of the cuffs the venous volume increases gradually. When maximum volume was achieved, release of cuff-pressure resulted in a volume decrease measured by plethysmography. The tangent to the slope of the volume decreased wais drawn $0.5 \mathrm{~s}$ after pressure release and the slope was converted into a venous emptying rate (VER). This procedure wais performed at five different cuff pressures (50-15 $\mathrm{mmHg}$ ). Subsequently the corresponding VER values were plotted against the effective cuff pressures. The slope of the line through the points gives an angle $\beta$. In analogy with Ohm's law the VOR was calculated as $1 /$ tangent $\beta$. The VOR value is expressed in resistance units: $(\mathrm{mmHg} \times \min ) / \%$. In a previous study a VOR exceeding $0.8 \mathrm{mmHg}{ }^{*} \mathrm{~min} / \%$ was considered abnormal. The nonthrombotic leg was always normal and used as control. The reproducibility of the VOR was within $10 \%$ [10].

Duplex examinations were performed by two experienced vascular technicians with a Toshiba SSA 270A scanner with a 3.75 or $5 \mathrm{MHz}$ probe in low flow setting. The examination was performed with the patient in a reverse Trendelenburg position at $45^{\circ}$, the knees flexed and with the feet resting on a foot-support. Sixteen Vein segments were examined: the common femoral vein, the femoral vein (proximal and middle), the great saphenous vein (proximal, middle and distal), the popliteal vein, the small saphenous vein, the posterior and anterior tibial veins, the peroneal and gastrocnemial veins. In the calf each artery is accompanied by 2 veins; these were numbered: 1 being the most superficial and 2 the deeper vein. The distal superficial femoral vein is often not compressible because of the anatomical position in the canal of Hunter. After the third month the peroneal, gastrocnemial veins and tibial anterior veins were not examined because many veins could not be traced and these measurements appeared very time-consuming. The results of the remaining 10 vein segments were used for analysis. The compressibility was assessed in the transverse plane with gentle pressure applied to the overlying skin by the duplexprobe. In the longitudinal plane the presence of the Doppler signal was measured. In the proximal veins a spontaneous Doppler signal is usually present; in the distal veins a flow signal was considered present after gentle squeezing the extremity below the level of measurement. The definition of the thrombosis score (TS) was based on the description of extent of thrombus and site involvement by Porter et al. [11]. A vein segment was considered occluded when the vein segment was non-compressible and no Doppler signal was found (TS = 2) and non-occluded if it was not completely compressible and flow was present (TS =1). A fully patent vein segment showed complete compressibility and flow $(\mathrm{TS}=0)$. TS values were calculated by counting the TS values of the 10 vein segments. The kappa-coefficient of the interobserver variability was 0.77 [12]. Reflux was measured in the longitudinal plane. In the proximal veins the Valsalva manoeuvre was used to provoke reflux and in the distal veins by manual compression with sudden release. Pathological reflux was defined as a reversed flow duration of more than $1 \mathrm{~s}$ in the proximal veins and more than $0.5 \mathrm{~s}$ in the distal veins. The kappa of the interobserver variability was 0.86 [12].

We performed a sample size calculation assuming a two-sided $P$-value of 0.05 and a power of $80 \%$ and SD of 3 to detect a two-point decrease of the Villalta-score. This corresponds with a decrease of PTS (Villalta score $>5$ ) from 25 to $15 \%$. This sample size calculation yielded a sample size of 36 patients in both groups. Intention to treat and on treatment analysis was performed.

\section{Results}

Sixty-nine patients were randomized to bandaging ( $n=34)$ or no bandaging $(n=35)$. Idiopathic DVT was present in 42 patients, in 27 patients DVT was secondary because of immobilisation $(n=11)$, recent trauma $(n=7)$ or operation $(n=9)$.

All patients were followed up for at least 12 months. During this period two patients in the bandage group died because of malignant disease, diagnosed after inclusion in the study. Two patients were lost to follow up (one in both groups). In the control group one patient was not included in the analysis after she was diagnosed with a non-Hodgkin lymphoma in her pelvis one week after inclusion. Table 1 shows the patient characteristics at baseline. Location and extension of thrombosis was equal in both groups. 
Table 1 Baseline characteristics of the patients (intention to treat)

\begin{tabular}{llll}
\hline & Bandaging & No bandaging & $P$-values \\
\hline Number (\%) & $31(48.4)$ & $33(51.6)$ & \\
Age (range) & $64(25-80)$ & $53(20-86)$ & 0.55 \\
Male/female & $15 / 16$ & $17 / 16$ & 0.80 \\
Location of DVT (\%) & & $5(16.1)$ & 0.79 \\
$\quad$ Distal & $4(12.9)$ & & \\
$\quad$ & & $11(33.3)$ \\
Proximal & $5(16.1)$ & $1(3.2)$ & \\
Fopliteal & $3(9.7)$ & $14(45.1)$ & \\
Popliteal and femoral & $13(41.9)$ & $2(6.5)$ & \\
Pelvic & $5(16.1)$ & $28(43.8)$ & \\
& $27(42.2)$ & $19(29.7)$ & \\
$\quad$ Left & $18(28.1)$ & $14(21.9)$ & 0.58 \\
$\quad$ Right & $13(20.3)$ & $1.75(1.53-2.03)$ & 0.57 \\
Length in m (range) & $1.72(1.51-1.90)$ & $76.5(53.0-164.0)$ & \\
Weight in kg (range) & $73.0(48.6-103.3)$ &
\end{tabular}

Improvement of symptoms was better in patients with compression bandages on day 7. All patients in the bandaging group had a decrease of symptoms, six patients in the no-bandaging group had an increase of symptoms. After 1 and 3 months symptoms were improved equally in both groups (Fig. 1). The difference in leg circumference of the thrombosed compared to the healthy leg decreased more rapidly in patients with bandages compared to patients without bandages on day 7 and 30 (resp day 7: 1.0 vs. $2.75 \mathrm{~cm}$ and day $30-0.5$ vs. $2 \mathrm{~cm}, P<0.05$ ) (Fig. 2). In seven patients without bandages edema was still present after 10 days and compression bandage was applied.

Table 2 shows the mean outcomes of the non-invasive examinations. It demonstrates that there were no differences in VOR, TS and reflux between the two groups.

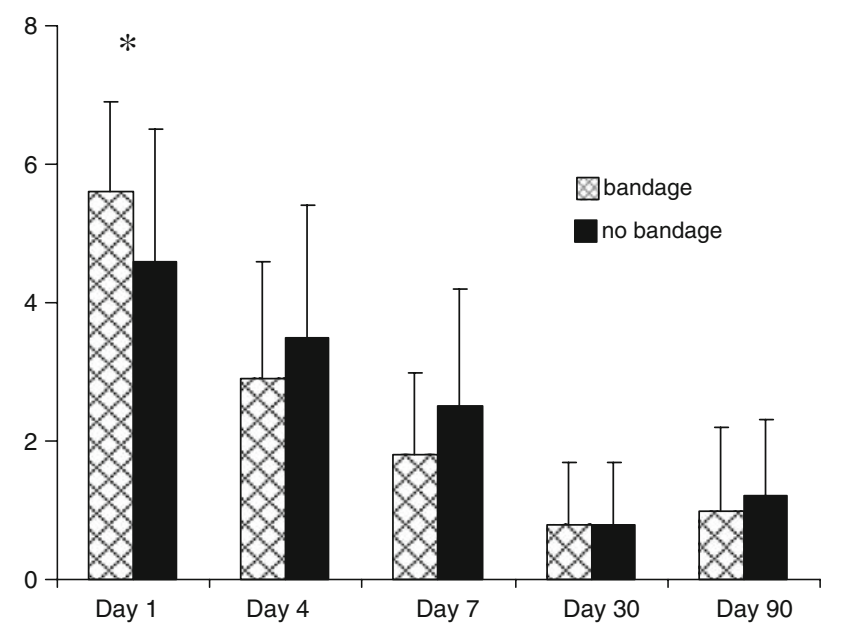

Fig. 1 Decrease of clinical symptoms with and without bandage

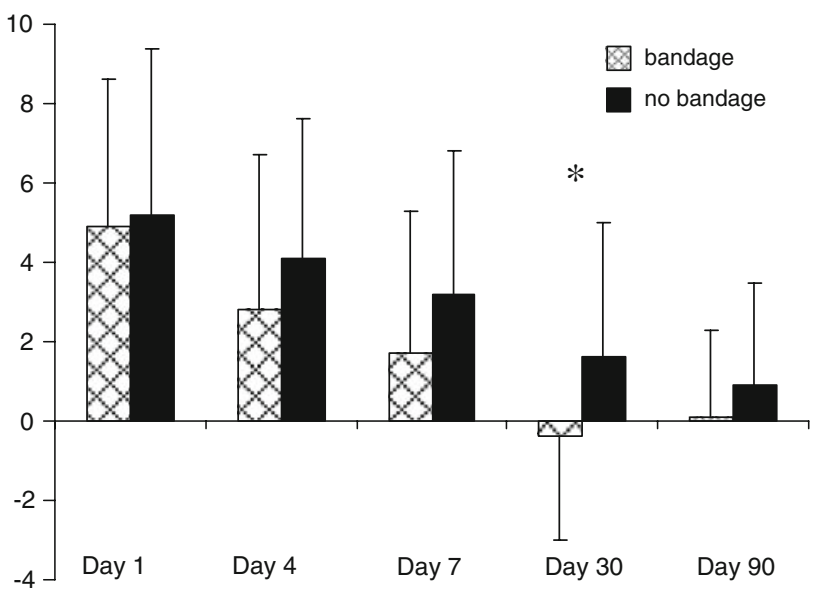

Fig. 2 Decrease of leg circumference in centimeters with and without bandage

Table 2 Mean $( \pm \mathrm{SD})$ outcomes of non-invasive venous examinations (intention to treat analysis)

\begin{tabular}{llll}
\hline & $\begin{array}{l}\text { Bandaging } \\
(N=31)\end{array}$ & $\begin{array}{l}\text { No bandaging } \\
(N=33)\end{array}$ & $P$ \\
\hline VOR & & & \\
Day 7 & $1.43( \pm 1.16)$ & $1.72( \pm 1.85)$ & 0.40 \\
Day 30 & $0.91( \pm 0.80)$ & $0.95( \pm 0.61)$ & 0.43 \\
Day 90 & $0.77( \pm 0.55)$ & $0.80( \pm 0.58)$ & 0.37 \\
Thrombosis score & & \\
Day 7 & $4.11( \pm 3.02)$ & $4.33( \pm 1.41)$ & 0.63 \\
Day 30 & $2.10( \pm 2.38)$ & $2.78( \pm 1.48)$ & 0.15 \\
Day 90 & $2.25( \pm 1.67)$ & $2.00( \pm 1.41)$ & 0.90 \\
Reflux & & & \\
Day 90 & $1.0( \pm 2.14)$ & $0.43( \pm 1.13)$ & 0.40 \\
\hline
\end{tabular}


Table 3 Clinical PTS after 1 year (intention to treat)

\begin{tabular}{lcclc}
\hline CEAP & $\begin{array}{l}\text { Bandaging } \\
(N=31)\end{array}$ & $\begin{array}{l}\text { No bandaging } \\
(N=33)\end{array}$ & RR & $95 \%$ CI \\
\hline 0 & 1 & 2 & & \\
1 & 14 & 12 & $0.92^{*}$ & $0.55-1.56$ \\
2 & 4 & 5 & $1.14^{* *}$ & $0.65-1.99$ \\
3 & 4 & 7 & & \\
4 & 8 & 7 & & \\
5 & 0 & 0 & & \\
6 & 0 & 0 & &
\end{tabular}

$*$ RR for PTS defined as CEAP $\geq 3$, ** RR for PTS defined as CEAP $\geq 4$

Table 3 demonstrates the classification according to the CEAP clinical scale and the Villalta score. The overall incidence of PTS, defined as CEAP $\geq 3$ was $39 \%$ in patients with bandages and $42 \%$ in patients without bandages $(\mathrm{RR}=0.92$, 95\% CI 0.55-1.56). When PTS was defined as CEAP $\geq 4$ RR was 1.14 (95\% CI 0.65-1.99). Using on treatment analysis RR for CEAP $\geq 3$ and CEAP $\geq 4$ were 1.04 and 1.09 respectively. The overall incidence of PTS, defined as Villalta $\geq 5$ was $29 \%$ in patients with bandages and $33 \%$ in patients without bandages (RR 0.87 , 95\% CI 0.41-1.8). Mean CEAP score after 1 year in patients without bandages was 2.15 , in patients with bandages 2.12, with a mean difference of 0.22 (95\% CI -0.64-0.68). Mean Villalta score after 1 year in patients without bandages was 4.09 , with bandages 3.74 , with a mean difference of 0.35 (95\% CI -1.47-2.16).

\section{Discussion}

There is a lack of consensus regarding the optimal timing of initiating of compression therapy in the treatment of DVT to prevent PTS. We studied the influence of immediate compression bandaging before application of elastic stockings in the acute phase of DVT. In the first week bandaging was effective in reducing edema and complaints, but after 1 and 3 months improvements were equal in both patients with or without bandages. There was no effect on thrombus regression, valve incompetence and the development of clinical PTS after 1 year.

In acute DVT pain results from an increase of compartmental pressure resulting from congestion of muscle veins by thrombi and trapped blood. External leg compression has several physical effects. It counteracts edema formation, leads to a narrowing of superficial and deep veins and increases blood flow velocity in the microcirculation. Partsch et al. demonstrated that compression therapy combined with ambulation leads to a faster reduction of leg swelling and pain compared to bedrest
[13]. They also showed that multilayer compression bandages with high stiffness applied with a pressure higher than $50 \mathrm{mmHg}$ have beneficial haemodynamic effects that cannot be achieved by elastic compression stockings with a maximum pressure of 30-40 $\mathrm{mmHg}[7,13]$. Since venous hypertension and valve damage occur soon after a thrombotic episode, it is suggested that compression therapy must be applied quickly, but it has never been investigated whether immediate compression therapy in the acute phase of DVT further reduces the incidence of clinical PTS.

We studied the influence of compression bandaging before application of elastic stockings in the acute phase of DVT. Patients in the no-bandaging group did not get compression therapy in the first 2 weeks, since it takes about two weeks to measure and produce sized-to-fit elastic stockings. Both groups received the elastic stockings after 7-14 days. Both groups were treated with anticoagulants with a similar INR, also all patients were equally mobile. Like the study of Partsch et al. improvement of symptoms and decrease in leg circumference was better in patients with compression bandages, although after 1 and 3 months symptoms improved equally in both groups.

There is no objective criterion standard test to diagnose PTS. For clinical investigation several scales have been developed. We used both the CEAP scale, which describes mainly chronic venous disorders, and the Villalta score which also takes into account subjective symptoms. There was no difference in clinical stages of PTS according to nor the CEAP classification neither the Villalta classification after 1 year. The incidence of PTS in our study did not differ from that observed in large series of patients reported previously [1].

Both VOR and duplex are non-invasive tests with a good interobserver variability and can easily be used in studies and usual patient care [12, 14]. Previously we demonstrated that the measurement of TS, VOR and reflux at three months follow-up can be used as a proxy or surrogate endpoint for PTS [2]. In the current study there was no difference in the decline of VOR en TS between both groups. This means that compression therapy in the acute phase does not induce better thrombus regression compared to only elastic stockings and has no influence on the amount of reflux.

This study has some limitations. Firstly randomization was not blind. However, the vascular technicians and the dermatologist who classified PTS after 1 year were not informed. Secondly, no objective, gold standard test to diagnose PTS exists. For this reason we used objective non-invasive techniques as VOR and duplex. The disadvantage of the CEAP classification is that it is rather static, description of changes over time is limited and subjective symptoms are not included [15]. For this reason we also used the Villalta score. Both PTS classification systems are 
reasonably correlated with the ambulant venous pressure (AVP) [16].

Finally seven patients of the no-bandaging group received bandaging after all because of persistent swelling and pain. For this reason both an on-treatment and an intention-to-treat analysis were performed, with same results.

Based on the results of our study immediate compression bandaging in the acute phase of DVT reduces pain and leg circumference significantly, but it has no additional value compared to delayed compression therapy with stockings concerning regression of thrombus, development of reflux and clinical PTS. Therefore, in practice, applying elastic stockings within about 2 weeks seems sufficient. Immediate compression therapy with multilayer bandaging should only be applied to patients with severe complaints or swelling of the thrombosed leg.

Open Access This article is distributed under the terms of the Creative Commons Attribution Noncommercial License which permits any noncommercial use, distribution, and reproduction in any medium, provided the original author(s) and source are credited.

\section{References}

1. Prandoni P, Lensing AW, Prins MH et al (2004) Below-knee elastic compression stockings to prevent the post-thrombotic syndrome: a randomized, controlled trial. Ann Intern Med 141:249-256

2. Roumen-Klappe EM, den Heijer M, Janssen MC, van d V, Thien T, Wollersheim H (2005) The post-thrombotic syndrome: incidence and prognostic value of non-invasive venous examinations in a six-year follow-up study. Thromb Haemost 94:825-830

3. Kahn SR, Ginsberg JS (2004) Relationship between deep venous thrombosis and the postthrombotic syndrome. Arch Intern Med 164:17-26

4. Kahn SR, M'Lan CE, Lamping DL, Kurz X, Berard A, Abenhaim LA (2004) Relationship between clinical classification of chronic venous disease and patient-reported quality of life: results from an international cohort study. J Vasc Surg 39:823-828

5. Kahn SR, M'Lan CE, Lamping DL, Kurz X, Berard A, Abenhaim L (2004) The influence of venous thromboembolism on quality of life and severity of chronic venous disease. J Thromb Haemost 2:2146-2151

6. Brandjes DP, Buller HR, Heijboer H et al (1997) Randomised trial of effect of compression stockings in patients with symptomatic proximal-vein thrombosis. Lancet 349:759-762

7. Blattler W, Partsch H (2003) Leg compression and ambulation is better than bed rest for the treatment of acute deep venous thrombosis. Int Angiol 22:393-400

8. Eklof B, Rutherford RB, Bergan JJ et al (2004) Revision of the CEAP classification for chronic venous disorders: consensus statement. J Vasc Surg 40:1248-1252

9. Klein Rouweler BJF, Brakkee AJM, Kuiper JP (1989) Plethysmographic measurement of venous flow resistance and venous capacity in the human leg. Part one: method. Phlebology 4:241250

10. Klein Rouweler BJF, Brakkee AJM, Kuiper JP (1989) Plethysmographic measurement of venous flow resistance and venous capacity in human legs. Part two: normal values related to age, sex and site of measurement. Phlebology 4:251-257

11. Porter JM, Moneta GL (1995) Reporting standards in venous disease: an update. International Consensus Committee on Chronic Venous Disease. J Vasc Surg 21:635-645

12. Haenen JH, van LH, Janssen MC et al (1999) Venous duplex scanning of the leg: range, variability and reproducibility. Clin Sci 96:271-277

13. Partsch H, Blattler W (2000) Compression and walking versus bed rest in the treatment of proximal deep venous thrombosis with low molecular weight heparin. J Vasc Surg 32:861-869

14. Haenen JH, Janssen MC, van LH et al (1998) Duplex ultrasound in the hemodynamic evaluation of the late sequelae of deep venous thrombosis. J Vasc Surg 27:472-478

15. Nicolaides AN (2000) Investigation of chronic venous insufficiency: a consensus statement. Circulation 102:E126-E163

16. Gabriel F, Labios M, Portoles $O$ et al (2004) Incidence of postthrombotic syndrome and its association with various risk factors in a cohort of Spanish patients after one year of follow-up following acute deep venous thrombosis. Thromb Haemost 92:328336 